\title{
Science needs management data for a better prediction of climate change effects on socio-ecosystems
}

\author{
Bruno Fady ${ }^{1}$ (D) Thomas Geburek $^{2} \cdot$ Ivan Scotti ${ }^{3}$ \\ Received: 27 November 2018 / Accepted: 17 December 2018 / Published online: 30 January 2019 \\ (C) INRA and Springer-Verlag France SAS, part of Springer Nature 2019 \\ Keywords Experimental approach $\cdot$ Data availability $\cdot$ Forest $\cdot$ Climate change $\cdot$ Infrastructure
}

Recent articles show that climate-change science lacks multiscale ecosystem demographics and dynamics data to test, improve, and validate its models and recommendations. This is a major drawback; one that leads to skepticism from managers, policymakers, and the public. It may be at least partially compensated if the scientific community has access to, and analyzes data from, natural habitat management, particularly forest management.

Data included in forest management records could significantly contribute to fill the gaps left by standard research programs, these days unavoidably restricted in scope, funding, and duration (typically 3 to 5 years). By contrast, forest management in, e.g., Japan, Europe, and North-America have kept track of many of their activities over very long periods of time, up to decades and even back to the nineteenth century in some cases.

Handling Editor: Erwin Dreyer

Contribution of the co-authors All co-authors contributed equally to the writing of the paper.

Bruno Fady

bruno.fady@inra.fr

1 INRA, URFM, Ecology of Mediterranean Forests, 84914 Avignon, France

2 BFW, Department of Forest Genetics, 1131 Vienna, Austria

3 INRA, URFM, Ecology of Mediterranean Forests, 84914 Avignon, France
Management actions (such as selection of species and seed sources, plantation, and thinning) influence the dynamics of forest ecosystems at multiple scales. Management regularly monitors its actions and their outcomes from the implementation stage onwards, using standardized procedures and protocols. Repeated thousands of times over different climate habitats, these outcomes constitute massive amounts of data that can be statistically analyzed and have far-reaching, general value. For example, how climate affects species adaptation processes could be drawn from the repeated failure or success of particular species, populations or genotypes in reforestation programs across entire continents and distribution areas.

Management data may be owned privately or publicly, and getting access to these long data series is the real challenge. To add insult to injury, management agencies have tended to stop archiving management data when data storage moved from the age of paper to the age of computers. Publicly owned data at least should be available to the scientific community and for public management agencies, making data available should become part of their mandate. This requires strong institutional support, which would be well received in an era of growing public and political interest for open data and open science.

Ground proofing management strategies (such as assisted migration and composite provenancing) would greatly benefit from access to, and the analysis of, forest management data (Figs. 1 and 2, both forest ecosystems are threatened by climate change). 


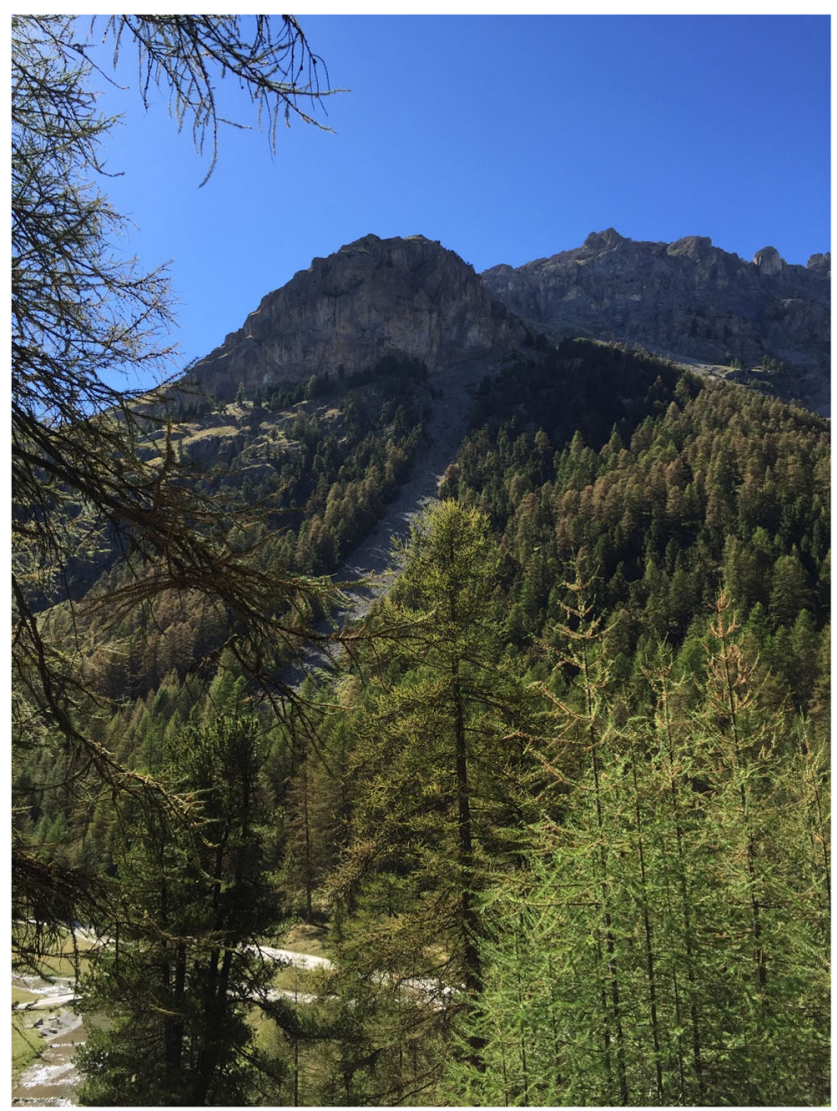

Fig. 1 The Boréon Pinus cembra/Larix decidua mixed forest in the southern French Alps

At a time when countries in Europe and worldwide are placing research infrastructures on their new roadmap and requesting that nature-based solutions be applied for

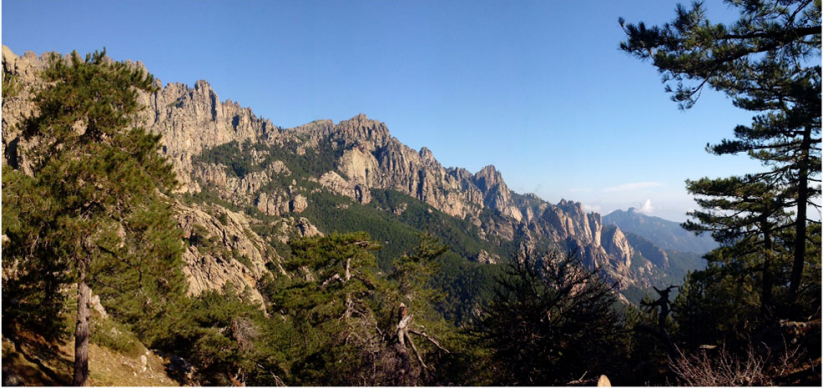

Fig. 2 Abies alba Punteniellu forest in Corsica, with black pines in the foreground

sustainable development, demanding that management data should continue to be collected and made openly accessible in the data archives of infrastructures would be more than sensible. Not only this relatively cost-limited engagement would contribute to bridging the gap between research and management, it would also truly benefit building groundproofed adaptation strategies under climate change.

\section{Compliance with ethical standards}

Conflict of interest The authors declare that they have no conflict of interest.

Publisher's note Springer Nature remains neutral with regard to jurisdictional claims inpublished maps and institutional affiliations. 